\begin{tabular}{|c|c|c|}
\hline \multirow{2}{*}{$\begin{array}{r}\text { Case Reports in } \\
\text { Gastroenterology }\end{array}$} & \multicolumn{2}{|c|}{ Case Rep Gastroenterol 2019;13:66-72 } \\
\hline & $\begin{array}{l}\text { DOI: 10.1159/000496917 } \\
\text { Published online: February 14, } 2019\end{array}$ & $\begin{array}{l}\text { ( } 2019 \text { The Author(s) } \\
\text { Published by S. Karger AG, Basel } \\
\text { www.karger.com/crg }\end{array}$ \\
\hline
\end{tabular}

\title{
Successful Resection of Solitary Abdominal Wall Metastasis of Sarcomatous Intrahepatic Cholangiocarcinoma
}

\author{
Kosuke Ono ${ }^{a}$ Michinori Hamaoka ${ }^{a}$ Hiroyuki Egia Tsuyoshi Kobayashi ${ }^{a}$ \\ Hiroyuki Tahara $^{a}$ Shintaro Kuroda ${ }^{a}$ Seiichi Shimizu ${ }^{a}$ Shoichiro Mukai ${ }^{a}$ \\ Masatoshi Kochi $^{\mathrm{a}}$ Noriyuki Shiroma ${ }^{\mathrm{b}}$ Koji Arihiro $^{\mathrm{b}}$ Hideki Ohdan $^{\mathrm{a}}$ \\ aDepartment of Gastroenterological and Transplant Surgery, Graduate School of \\ Biochemical \& Health Sciences, Hiroshima University, Hiroshima, Japan; ${ }^{b}$ Department of \\ Anatomical Pathology, Hiroshima University Hospital, Hiroshima, Japan
}

\section{Keywords}

Cholangiocarcinoma $\cdot$ Resection $\cdot$ Metastasis

\begin{abstract}
Sarcomatous intrahepatic cholangiocarcinoma (ICC) is a rare histological variant of ICC that is composed of both adenocarcinoma (ICC component) and sarcomatous components. Surgery is believed to be the primary treatment, and some reports describe primary resection. However, due to the aggressive malignancy of sarcomatous ICC, there is no report regarding resection of a metastatic lesion. In this report, we present the case of a 75-year-old woman admitted to our hospital with the chief complaint of weight loss. Various imaging techniques demonstrated a single mass in the liver and cecum. A cecal gastrointestinal stromal tumor accompanied by liver metastasis was suspected, and ileocecal resection was performed for diagnostic purposes. However, the tumor was present in the abdominal wall rather than in the cecum. The tumor was resected and diagnosed as undifferentiated sarcoma. We suspected the liver tumor was a series of lesions, so we performed hepatectomy. As the tumor was composed of both adenocarcinoma and sarcomatous components, it was diagnosed as sarcomatous ICC. The histological findings of the abdominal wall tumor were similar to those of sarcomatous ICC, so we
\end{abstract}




\section{Case Reports in Gastroenterology}

Case Rep Gastroenterol 2019;13:66-72

DOI: $10.1159 / 000496917$

c) 2019 The Author(s). Published by S. Karger AG, Basel www.karger.com/crg

Ono et al.: Resection of Solitary Abdominal Wall Metastasis

diagnosed the abdominal wall tumor as a solitary metastasis of sarcomatous ICC. In this case, solitary metastasis was observed, and we were able to resect both the primary and metastatic lesions. This case illustrates that when solitary metastasis can be seen in sarcomatous ICC, radical resection is possible.

\section{Introduction}

Epithelial tumors with sarcomatous components are occasionally seen in various organs, including the liver. Most sarcomatous carcinomas in the liver are thought to be sarcomatous hepatocellular carcinoma [1]. Recently, there have been some reports demonstrating sarcomatous changes in cholangiocarcinoma. In the WHO classification of tumors, this type of tumor is defined as sarcomatous intrahepatic cholangiocarcinoma (sarcomatous ICC). The sarcomatous component of the tumor microscopically resembles sarcoma, but the expression of both epithelial and mesenchymal features is characteristic [2]. The prognosis of sarcomatous ICC is reportedly worse than that of ordinary ICC, because of its aggressive malignancy [3]. Surgery is believed to be the primary treatment for sarcomatous ICC, and some reports describe primary resection in the case of local progression. However, in cases with metastasis, the tumor is found in an unresectable state such as in multiple metastases or peritoneal dissemination. Accordingly, to the best of our knowledge, there is no report regarding resection of a metastatic lesion. We herein report the successful resection of a primary lesion and solitary abdominal wall metastasis of sarcomatous ICC.

\section{Case Report}

A 75-year-old woman was admitted to our hospital with the chief complaint of weight loss; she had lost $3 \mathrm{~kg}$ of body mass in the previous 2 months. There was no past history of liver or biliary disease. A physical examination revealed the following: height, $150.0 \mathrm{~cm}$; body weight, $42.0 \mathrm{~kg}$; and body mass index, $18.7 \mathrm{~kg} / \mathrm{m}^{2}$. The main laboratory data were as follows: hemoglobin, $11.1 \mathrm{~g} / \mathrm{dL}$; white blood cells, $5.63 \times 10^{3} / \mu \mathrm{L}$; platelets, $271 \times 10^{3} / \mu \mathrm{L}$; total bilirubin, $0.5 \mathrm{mg} / \mathrm{dL}$; aspartate aminotransferase, $15 \mathrm{IU} / \mathrm{L}$; alanine aminotransferase, $10 \mathrm{IU} / \mathrm{L}$; carcinoembryonic antigen, $1.1 \mathrm{IU} / \mathrm{mL}$; and carbohydrate antigen 19-9, $2.0 \mathrm{IU} / \mathrm{mL}$.

A computed tomography scan revealed a tumor $5 \mathrm{~cm}$ in size with irregular enhancement in the ileocecal region and a tumor $6.5 \times 4 \mathrm{~cm}$ in size with ring enhancement in segment 8 of the liver (Fig. 1a). Colonoscopy revealed a $4-\mathrm{cm}$ lesion suspected of being a submucosal tumor in the ileocecal region (Fig. 1b). An endoscopic biopsy was performed, but confirmation was difficult due to too little tissue. A cecal gastrointestinal stromal tumor accompanied by liver metastasis was suspected, and ileocecal resection was planned for diagnostic purposes.

Intraoperative examination revealed that the tumor was present on the abdominal wall and pressed the cecum, but there was no invasion (Fig. 2a). The parietal peritoneum was dissected to secure the stump, and the tumor was excised. The tumor measured $55 \times 40 \times 38 \mathrm{~mm}$ (Fig. 2b). Histological examination showed diffuse growth of large spindle cells (Fig. 2c). Immunohistochemically, the tumor reacted positively to both cytokeratin and vimentin, but did not react to DOG1, S100, or antibodies, suggesting a lymphoma (Fig. 2d). From these findings, the tumor was diagnosed as undifferentiated sarcoma.

We suspected the liver tumor was a series of lesions, so we performed hepatectomy. The tumor was present in liver segment 8 , and it was present on the liver surface and invaded part 
of the diaphragm and the lower lobe of the right lung. Subsegmentectomy of liver segment 8 and partial resection of the right diaphragm and right lung were performed. Macroscopically, the tumor specimen measured $7 \times 6 \times 5 \mathrm{~cm}$ (Fig. 3a), and the tumor invaded the diaphragm and lung. Microscopically, the tumors were mostly composed of pleomorphic cells and spindle cells. Moreover, a ductogenic image was observed in the tumor. Vascular invasion was not observed (Fig. 3b). Upon immunohistochemical staining, the tumor reacted positively to cytokeratin-7, EMA, and HHF35, but negatively to hepatocytes and c-Kit (Fig. 3c).

As the tumor was composed of both adenocarcinoma and sarcomatous components, it was diagnosed as sarcomatous ICC. The histological findings of the abdominal wall tumor were similar to those of sarcomatous ICC. From these findings, we diagnosed the abdominal wall tumor as a solitary metastasis of sarcomatous ICC.

\section{Results}

The patient's postoperative course was uneventful, and she was discharged on the 15th postoperative day. Five months after surgery, a CT scan revealed no sign of recurrence.

\section{Discussion}

In this report, we demonstrated that solitary metastasis can be seen in sarcomatous ICC and that, in this situation, radical resection is possible. The prevalence of sarcomatous ICC is reported to be $4.5 \%$ of all ICC [4]. Furthermore, the prognosis for sarcomatous ICC is reportedly worse than that for ordinary ICC [3]. One factor concerning the poor prognosis is the tendency of symptoms to occur at a later stage of the disease, at which time radical resection is often impossible when a tumor is found [5]. Another factor is the aggressive intrahepatic spreading and frequent metastasis of sarcomatous cells [6]. Some reports demonstrate that radical resection is a strong option for treating this type of tumor $[3,7,8]$. For instance, Kaibori et al. [3] showed that the survival rate of patients with surgical resection was significantly higher than that of patients without surgical resection. Moreover, Watanabe et al. [8] showed that the prognosis for patients receiving surgery was better than that for patients with ordinary ICC without surgery.

To the best of our knowledge, in the case of multiple metastases and peritoneal dissemination, there is no report of the metastasis being resected. Therefore, although it is unknown whether metastatic lesion resection contributes to prognosis, a better prognosis is expected compared to that for cases wherein the metastasis could not be resected. Against such a background, knowledge regarding successful resection of solitary metastasis, as in the current case, is valuable. The reason why this tumor spread solitarily to the abdominal wall is unclear. Hematogenous metastasis is suspected, because the metastatic lesion was solitary and there was no disseminated lesion in the peritoneal cavity. The primary lesion was exposed outside the serosal membrane and invaded the right diaphragm; therefore, we speculate that the tumor metastasized from the vessel of the right peritoneum.

Also interesting in this case is that metastatic lesion resection preceded primary resection. In this case, we considered the abdominal wall tumor as a cecal gastrointestinal stromal tumor, so we resected the metastatic lesion first. Sarcomatous components are reported to be able to spread to the whole body [6]. Thus, such a case wherein the tumor with sarcomatous component is resected and a liver tumor also coexists, solitary abdominal wall metastasis of 
sarcomatous ICC should be considered, and curative resection should be performed if possible. In the present case, it was possible to perform radical resection including metastatic lesion, but careful follow-up is necessary. This is because some reports showed early recurrence, even in patients with radical resection of the primary tumor $[9,10]$. Although some reports showed the utility of chemotherapy, there is no clear information regarding optimal adjunctive treatment after initial surgical resection $[11,12]$. In the present case, we decided to conduct careful follow-up observation without chemotherapy.

In conclusion, solitary metastasis can be seen in sarcomatous ICC, and resection of the metastasis may precede primary resection. Radical resection may improve prognosis. Accordingly, such a case wherein the sarcomatous component is resected and a liver tumor coexists, solitary metastasis of sarcomatous ICC should be considered, and curative resection should be performed if possible.

\section{Statement of Ethics}

All procedures were performed in accordance with the ethical standards of the responsible committee on human experimentation (institutional and national) and with the Helsinki Declaration of 1975, as revised in 2008. The patient was not required to give informed consent for this case report because institutional review board approval was not required for a retrospective analysis of one case.

\section{Disclosure Statement}

The authors have no conflicts of interest to disclose.

\section{Author Contributions}

K. Ono and M. Hamaoka wrote the manuscript; all other authors contributed equally to medical treatment.

\section{References}

1 Maeda T, Adachi E, Kajiyama K, Takenaka K, Sugimachi K, Tsuneyoshi M. Spindle cell hepatocellular carcinoma. A clinicopathologic and immunohistochemical analysis of 15 cases. Cancer. 1996 Jan;77(1):51-7.

2 Kwon JH, Kang YN, Kang KJ. Carcinosarcoma of the liver: a case report. Korean J Radiol. 2007 Jul-Aug; 8(4):343-7.

3 Kaibori M, Kawaguchi Y, Yokoigawa N, Yanagida H, Takai S, Kwon AH, et al. Intrahepatic sarcomatoid cholangiocarcinoma. J Gastroenterol. 2003;38(11):1097-101.

4 Nakajima T, Tajima Y, Sugano I, Nagao K, Kondo Y, Wada K. Intrahepatic cholangiocarcinoma with sarcomatous change. Clinicopathologic and immunohistochemical evaluation of seven cases. Cancer. 1993 Sep;72(6):1872-7.

5 Tsou YK, Wu RC, Hung CF, Lee CS. Intrahepatic sarcomatoid cholangiocarcinoma: clinical analysis of seven cases during a 15-year period. Chang Gung Med J. 2008 Nov-Dec;31(6):599-605.

6 Sasaki M, Nakanuma Y, Nagai Y, Nonomura A, Barwick K. Intrahepatic cholangiocarcinoma with sarcomatous transformation: an autopsy case. J Clin Gastroenterol. 1991 Apr;13(2):220-5.

7 Shimada M, Takenaka K, Rikimaru T, Hamatsu T, Yamashita Y, Kajiyama K, et al. Characteristics of sarcomatous cholangiocarcinoma of the liver. Hepatogastroenterology. 2000 Jul-Aug;47(34):956-61. 
Ono et al.: Resection of Solitary Abdominal Wall Metastasis

8 Watanabe G, Uchinami H, Yoshioka M, Nanjo H, Yamamoto Y. Prognosis analysis of sarcomatous intrahepatic cholangiocarcinoma from a review of the literature. Int J Clin Oncol. 2014;19(3):490-6.

9 Inoue Y, Lefor AT, Yasuda Y. Intrahepatic cholangiocarcinoma with sarcomatous changes. Case Rep Gastroenterol. 2012 Jan;6(1):1-4.

10 Haratake J, Yamada H, Horie A, Inokuma T. Giant cell tumor-like cholangiocarcinoma associated with systemic cholelithiasis. Cancer. 1992 May;69(10):2444-8.

11 Malhotra S, Wood J, Mansy T, Singh R, Zaitoun A, Madhusudan S. Intrahepatic sarcomatoid cholangiocarcinoma. J Oncol. 2010;2010:701476.

12 Jung GO, Park DE, Youn GJ. Huge subcapsular hematoma caused by intrahepatic sarcomatoid cholangiocarcinoma. Korean J Hepatobiliary Pancreat Surg. 2012 May;16(2):70-4.
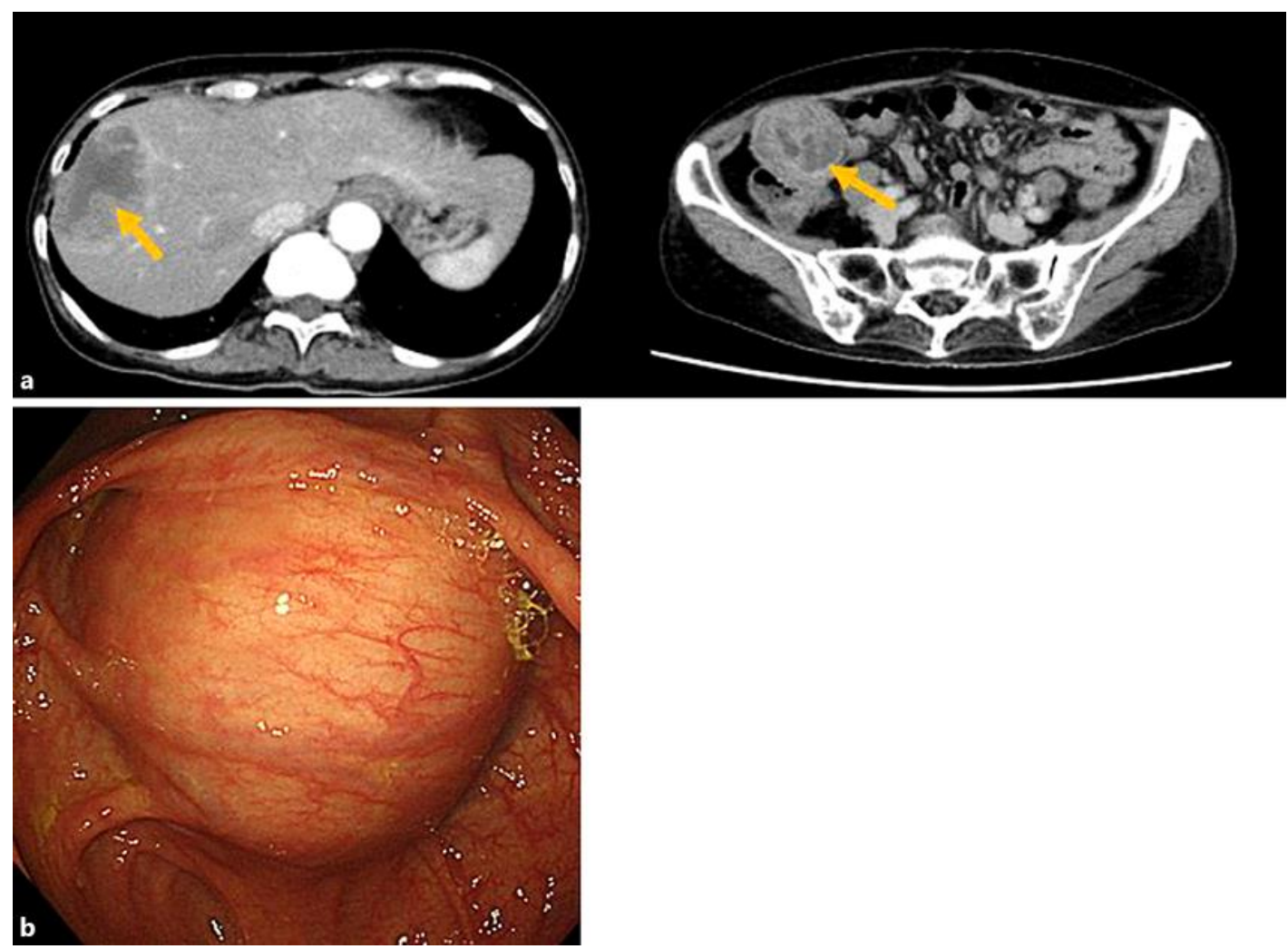

Fig. 1. a Computed tomography scans showing an irregularly enhanced mass, $5 \mathrm{~cm}$ in diameter, in the ileocecal region (left, arrow) and a ring enhanced mass, $6.5 \mathrm{~cm}$ in diameter, in segment 8 of the liver (right, arrow). b Colonoscopy showed a 4-cm lesion suspected of being a submucosal tumor in the ileocecal region. 


\section{Case Reports in Gastroenterology}

\begin{tabular}{l|l}
\hline Case Rep Gastroenterol 2019;13:66-72 \\
\hline DOI: 10.1159/000496917 & $\begin{array}{l}\text { ( ) 2019 The Author(s). Published by S. Karger AG, Basel } \\
\text { www.karger.com/crg }\end{array}$ \\
\hline
\end{tabular}

Ono et al.: Resection of Solitary Abdominal Wall Metastasis

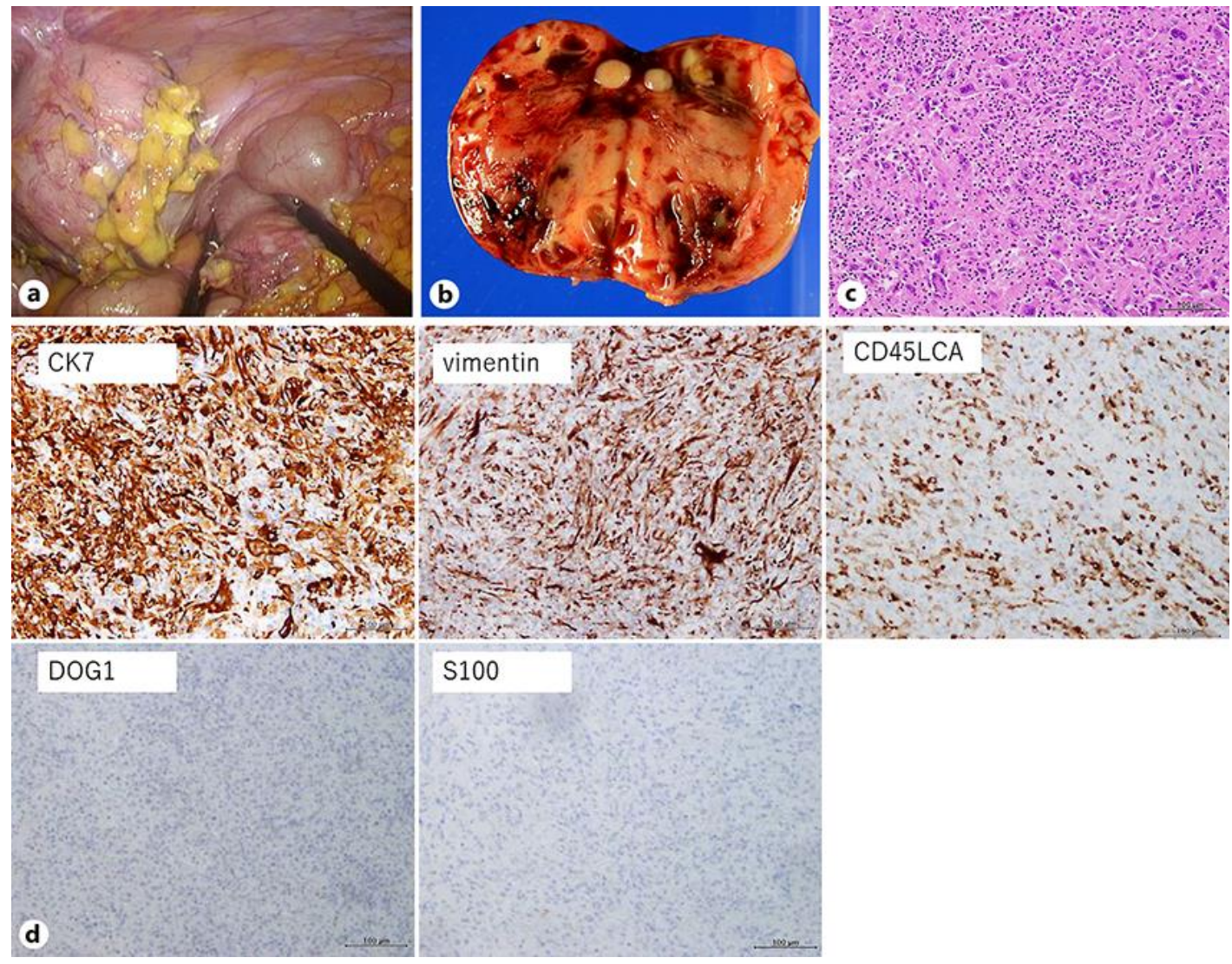

Fig. 2. a The tumor was present on the abdominal wall and pressed the cecum. $\mathbf{b}$ The tumor measured $5.5 \times 4.0 \times 3.8 \mathrm{~cm}$. c Histological examination showed diffuse growth of large spindle cells. HE. $\times 200$. d Immunohistochemically, the tumor reacted positively to both cytokeratin (CK7) and vimentin, but did not react to DOG1, S100, or antibodies (CD45LCA), suggesting a lymphoma. 


\section{Case Reports in Gastroenterology}

\begin{tabular}{l|l}
\hline Case Rep Gastroenterol 2019;13:66-72 \\
\hline DOI: 10.1159/000496917 & $\begin{array}{l}\text { @ 2019 The Author(s). Published by S. Karger AG, Basel } \\
\text { www.karger.com/crg }\end{array}$ \\
\hline
\end{tabular}

Ono et al.: Resection of Solitary Abdominal Wall Metastasis
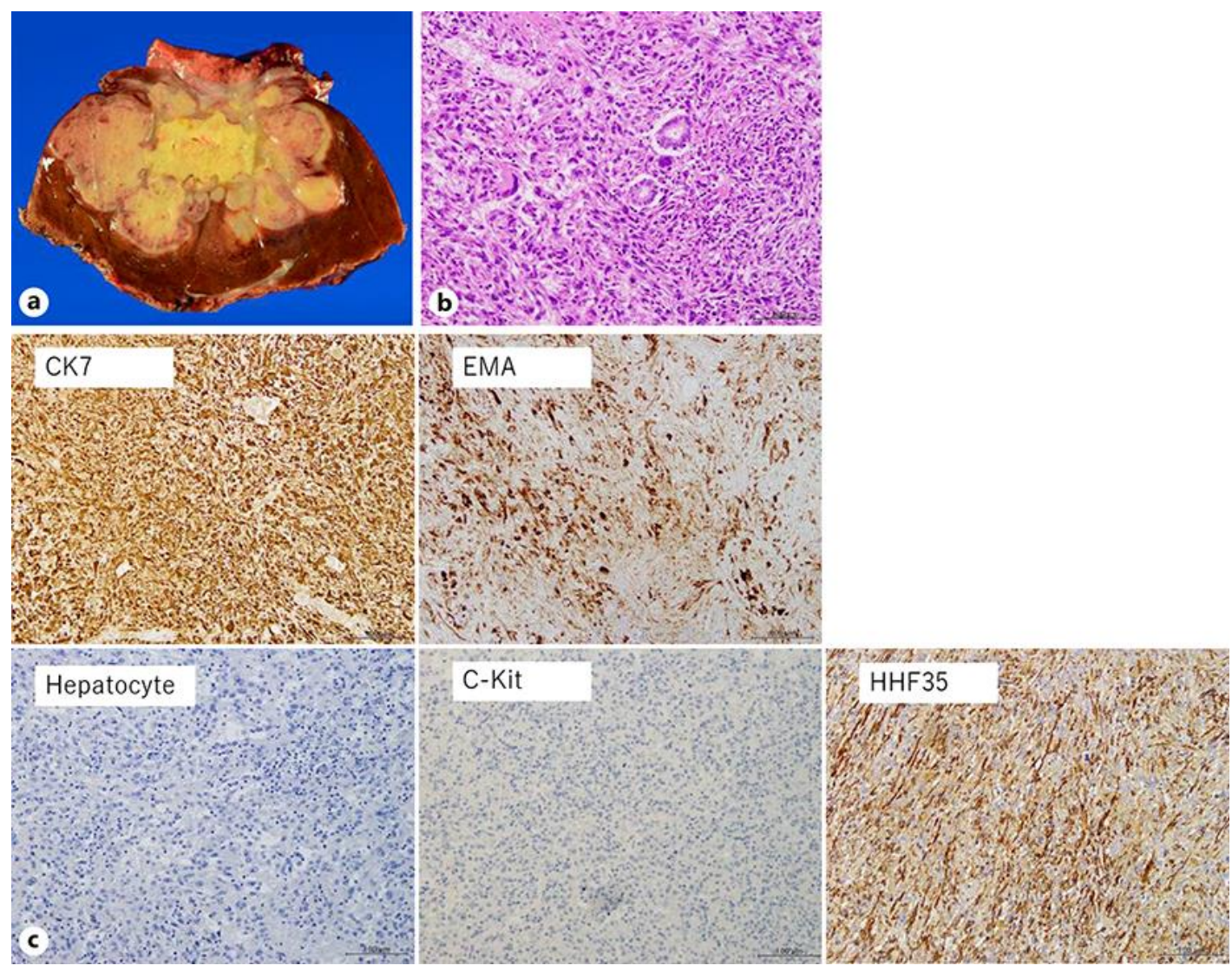

Fig. 3. a The tumor measured $7 \times 6 \times 5 \mathrm{~cm}$. b The tumor was composed of both adenocarcinoma and sarcomatous components. A ductogenic image was observed in the tumor. HE. $\times 200$. c On immunohistochemical staining, the tumor reacted positively to cytokeratin-7 (CK7), EMA, and HHF35, but negatively to hepatocytes and c-Kit. 